\title{
Limb-girdle muscular dystrophy 2l: phenotypic variability within a large consanguineous Bedouin family associated with a novel FKRP mutation
}

\author{
Tamar Harel $^{1}$, Yael Goldberg ${ }^{2}$, Stavit A Shalev ${ }^{2}$, Ilana Chervinski ${ }^{2}$, Rivka Ofir ${ }^{1}$ and \\ Ohad S Birk*,1,3
}

\begin{abstract}
${ }^{1}$ Laboratory of Human Molecular Genetics, Faculty of Health Sciences, Ben-Gurion University of the Negev, Beer-Sheva, Israel; ${ }^{2}$ Genetics Institute, Ha-Emek Medical Center, Afula, Israel; ${ }^{3}$ Genetics Institute, Soroka University Medical Center, Beer-Sheva, Israel
\end{abstract}

Limb-girdle muscular dystrophies (LGMDs) represent a group of diseases characterized mainly by muscle wasting of the upper and lower limbs, with a wide range of clinical severity. The clinical heterogeneity is paralleled by molecular heterogeneity; each of the $\mathbf{1 0}$ forms of autosomal-recessive LGMD recognized to date is caused by mutations in a distinct gene. In a large consanguineous Bedouin tribe living in northern Israel, 15 individuals affected by LGMD demonstrate an autosomal recessive pattern of inheritance. A genome-wide screen followed by fine mapping in this family revealed linkage to a region on chromosome 19 harboring the fukutin-related protein gene (FKRP), with a maximal LOD score of 4.8 for D19S902. FKRP, encoding a putative glycosyltransferase, has been implicated in causing congenital muscular dystrophy 1C (MDC1C), and has recently been shown to be mutated in LGMD2I. We identified a novel missense mutation in exon 4 of the FKRP gene in all the patients studied. Although all affected individuals were homozygous for the same mutation, a marked phenotypic variability was apparent within the family. This finding may suggest a role of modifier genes and environmental factors in LGMD2I. Moreover, the demonstration that an identical, novel mutation in the FKRP gene can cause a muscle disease of either a congenital onset or of a later onset within a single family provides clinical support to the molecular evidence, suggesting that MDC1C and LGMD2I are overlapping ends of one and the same entity. European Journal of Human Genetics (2004) 12, 38-43. doi:10.1038/sj.ejhg.5201087

Published online 1 October 2003

Keywords: LGMD; autosomal recessive; linkage analysis; intrafamilial variability

Introduction

Limb-girdle muscular dystrophies (LGMDs) are a group of muscle diseases whose mode of inheritance may be either autosomal dominant (LGMD type 1) or autosomal recessive (LGMD type 2). LGMDs (OMIM 601173) are characterized by a primary and progressive muscle degeneration of the shoulder and pelvic girdles, with a

*Correspondence: Dr O Birk, Genetics Institute, Soroka University Medical Center, POB 151, Beer-Sheva 84101, Israel. Tel: + 97258 795930; Fax: + 9728 6400042; E-mail: obirk@bgumail.bgu.ac.il

Received 3 March 2003; revised 19 June 2003; accepted 18 July 2003 wide range of clinical severity. ${ }^{1}$ The clinical heterogeneity of LGMD is paralleled by molecular heterogeneity. In all, 10 forms of LGMD type 2 have been mapped to date, and the specific gene defects have been identified in all: LGMD2A (CAPN3 at 15q15.1), ${ }^{2,3}$ LGMD2B (DYSF at 2p12-16), ${ }^{4,5}$ LGMD2C (SGCG at 13q12), ${ }^{6,7}$ LGMD2D (ADL at 17q12-q21.33), ${ }^{8-10}$ LGMD2E (SGCB at 4q12), ${ }^{11,12}$ LGMD2F (SGCD at 5q33-34), ${ }^{13,14}$ LGMD2G (TCAP at 17q11-q12), ${ }^{1,15}$ LGMD2H (TRIM32 at 9q31q34.1), ${ }^{16,17}$ LGMD2I (FKRP at 19q13.3), ${ }^{18,19}$ and LGMD2J (TTN at 2q31). ${ }^{20}$ The involved genes encode either structural components of muscle, that is, $\gamma_{--}, \alpha-, \beta-$, and 
$\delta$-sarcoglycans, responsible for LGMD2C to $2 \mathrm{~F}$, respectively, or proteins of other functions.

The fukutin-related protein gene (FKRP; OMIM 606596) is implicated in causing both LGMD2I (OMIM 607155) and congenital muscular dystrophy 1C (MDC1C) (OMIM 606612). Individuals in 17 LGMD2I families studied by Brockington et al $^{19}$ were found to carry mutations in FKRP. The FKRP transcript is expressed predominantly in the skeletal muscle, placenta, and heart. ${ }^{21}$

Abnormalities in the expression pattern of the $\alpha$-dystroglycan protein have been demonstrated in muscle biopsies of LGMD2I patients, ${ }^{19}$ indirectly suggesting that FKRP, a putative glycosyltransferase, is required for the post-translational modification of dystroglycan. FKRP is targeted to the medial-Golgi apparatus through its $\mathrm{N}$-terminus and transmembrane domains. Mutations in the putative active site of the protein or in the Golgi-targeting sequence are likely to cause mislocalization of the mutant protein in the cell and aberrant processing of dystroglycan, leading to MDC1C or LGMD2I. ${ }^{22}$

In a large consanguineous Bedouin tribe living in northern Israel, 15 individuals were found to be affected by a form of LGMD. The analysis of the pedigree demonstrated an autosomal recessive pattern of inheritance (Figure 1). A genome-wide linkage analysis, followed by fine-mapping and mutation analysis, revealed a novel mutation in the FKRP gene of the patients studied. The affected patients were all homozygous for the same mutation; albeit, considerable phenotypic variability was noted among them.

\section{Materials and methods} Patients

A total of 11 affected individuals, with a clinical diagnosis of LGMD, and eight unaffected individuals, from the same consanguineous Bedouin tribe, were subject to genetic analysis after informed consent. The clinical information on nine patients was gathered from existing medical records; selected patients were also available for present clinical assessment. The research protocol was approved by the local Internal Review Board of the Ben-Gurion University Faculty of Health Sciences.

\section{Linkage and haplotype analysis}

Genomic DNA was extracted from whole blood using standard procedures. The genome-wide linkage analysis was undertaken on DNA samples, using the ABI PRISM Linkage Mapping Set MD10 (Applied Biosystems). In all, 400 fluorescent-labeled microsatellite markers, spaced at approximately $10 \mathrm{cM}$ intervals, were amplified from genomic DNA by PCR, according to the manufacturer's instructions. The products were electrophoresed on an ABI PRISM 377 DNA Sequencer (Applied Biosystems) and analyzed using GeneScan software.

Fine mapping was carried out using markers D19S908 and D19S902, respectively, positioned at 69.50 and $72.72 \mathrm{cM}$ on the Marshfield map. PCR products were separated on a $6 \%$ polyacrylamide gel and visualized by silver staining. ${ }^{23}$ Haplotypes were manually constructed and analyzed.

LOD scores were computed for each marker at various recombination fractions $(\theta)$, using the maximum-likelihood estimation method.

\section{FKRP mutational analysis}

The 1.7-kb fragment containing the FKRP coding sequence $^{21}$ was amplified from genomic DNA, using primers designed by the following program: http://www.genome. wi.mit.edu/cgi-bin/primer/primer3.cgi.

FKRP 1F (5'-agc tgt aga aag ggg caa ggc-3'); FKRP 2R $\left(5^{\prime}\right.$-acc aga cgt gcg ctt cct gcg- $\left.3^{\prime}\right) ;$ FKRP 3F (5'-gtg gcc cta gta

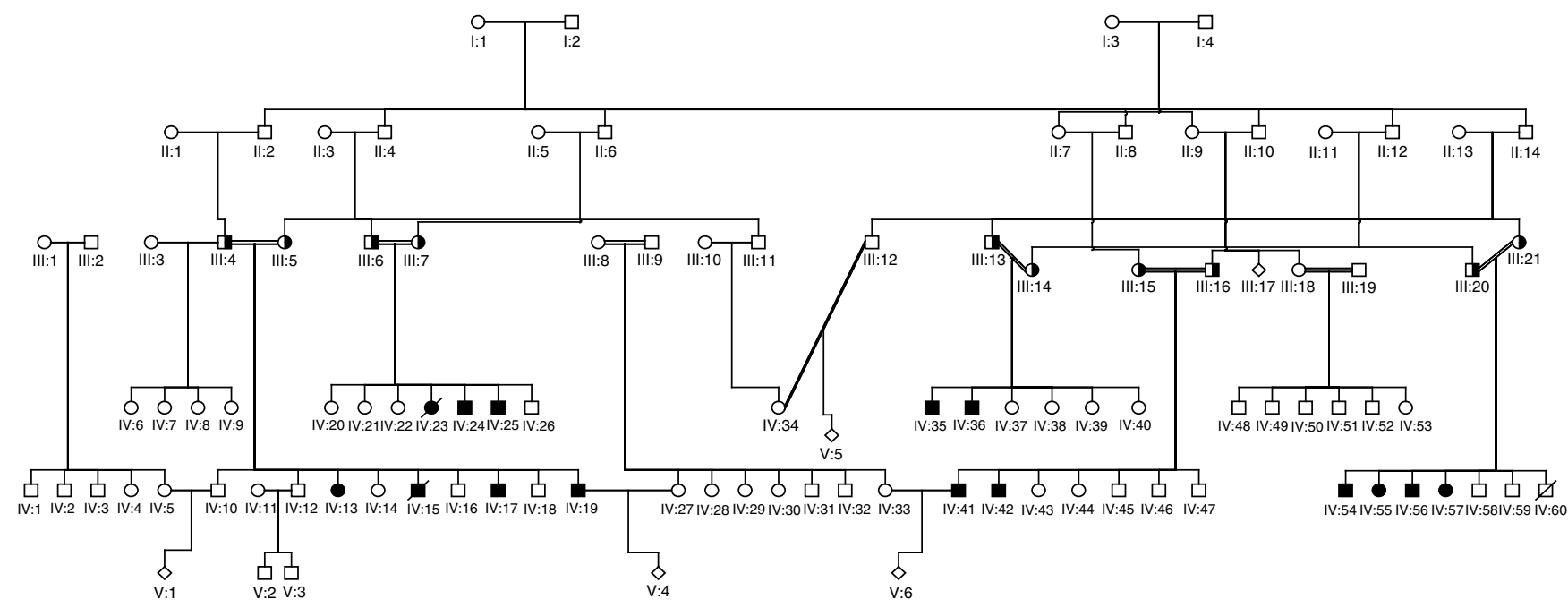

Figure 1 Pedigree of the investigated family. In total, 11 affected individuals were available for genetic analysis. The high rate of consanguinity and the autosomal recessive pattern of inheritance can be observed. 
Table 1 Summary of the features of the nine patients available for clinical studies

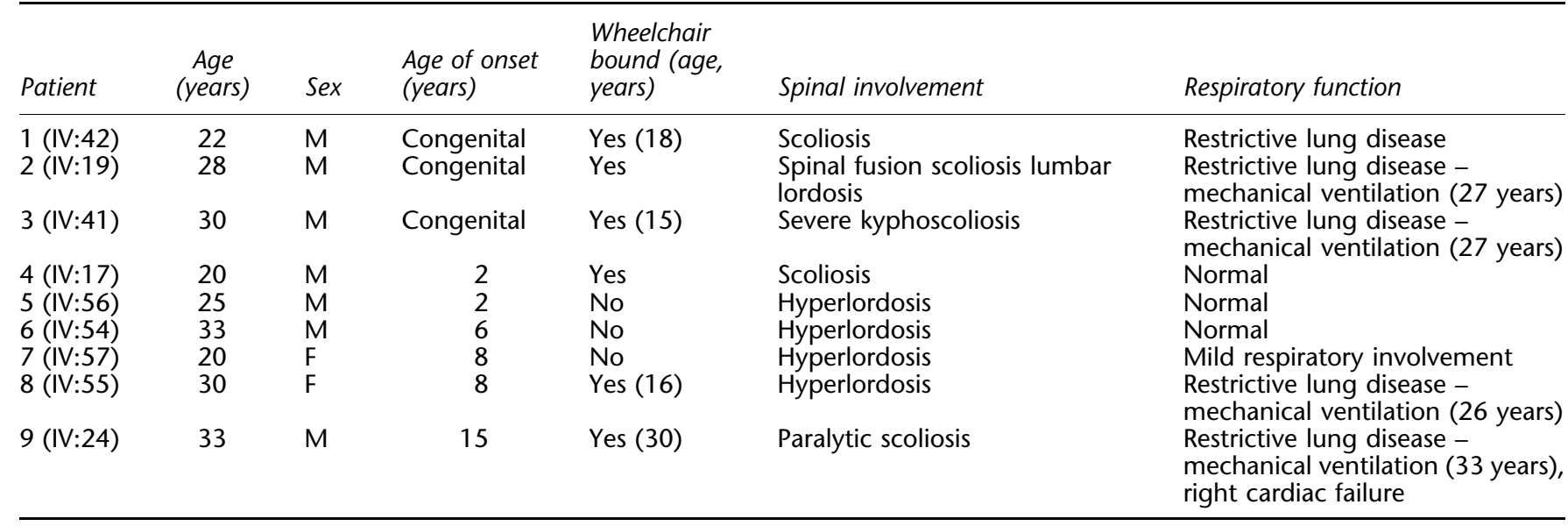

A correlation can be seen between the severity of the muscular involvement of the limbs (ie loss of ambulation) and the respiratory involvement.

cct gat gg-3'); FKRP 4R (5'-ctc ctc gta gag gta ggc gg- $\left.3^{\prime}\right)$; FKRP 5F (5'-ctc tac gag gag cgc tgg acg c-3'); and FKRP 6R $\left(5^{\prime}\right.$-gct cac aca gag ctt ctc ca- $\left.3^{\prime}\right)$.

PCR was performed in a total reaction volume of $50 \mu \mathrm{l}$ with $60 \mathrm{ng}$ of genomic DNA as the template and $2 \mathrm{U}$ Taq polymerase (QIAGEN). The amplification conditions were as follows: initial denaturation at $95^{\circ} \mathrm{C}$ for $10 \mathrm{~min}$, followed by 35 cycles of $94^{\circ} \mathrm{C}, 60^{\circ} \mathrm{C}$ (primers $3 \mathrm{~F} / 4 \mathrm{R}$ and $5 \mathrm{~F} / 6 \mathrm{R}$ ) or $65^{\circ} \mathrm{C}$ (primers $1 \mathrm{~F} / 2 \mathrm{R}$ ), and $72^{\circ} \mathrm{C}$, for $45 \mathrm{~s}$ each, and a final extension step at $72^{\circ} \mathrm{C}$ for $10 \mathrm{~min}$.

The PCR products were subject to agarose-gel electrophoresis and gel extraction (QIAGEN), followed by sequencing with either the forward or reverse primer on an ABI PRISM 377 DNA Sequencer (Applied Biosystems). The results were analyzed using Chromas software, and the DNA sequences obtained were compared to NCBI's 'Homo sapiens FKRP mRNA' (gi: 13236527), or to 'NT_011166.7' (gi: 17482678), by pairwise BLAST.

Normal population screening for the C160T mutation Genomic DNA was extracted from whole blood of 100 Bedouin individuals, unrelated to the tribe under study. Mutation analysis of the sequence amplified by primers FKRP $1 \mathrm{~F}$ and FKRP 2R was performed, as previously described. Alternatively, the PCR amplification products between primers FKRP 7F ( $5^{\prime}$-cat cac cct caa cct tct ggt cc- $\left.3^{\prime}\right)$ and FKRP 8R (5'-gcg ttg tca at gcc tcg aac tcc c- $\left.3^{\prime}\right)$ were subject to cleavage by the restriction enzyme AciI (BioLabs). Differential cleavage products of the wild-type versus mutant allele were analyzed on $2 \%$ agarose gels.

\section{Results}

Patients: clinical review

Nine of the patients were available for clinical studies. Notable intrafamilial phenotypic variability existed among these patients, in the following parameters: age of onset, pace of progression, extent of limb weakness and disability, respiratory involvement, and life expectancy (Table 1). No evidence of muscular impairment was manifested by heterozygotes in the family.

The age of onset of LGMD varied widely from birth to the second decade. All patients attained walking, although several had delayed motor milestones and minor symptoms during early childhood (ie, difficulty in running and climbing stairs, recurrent falls, or instability). Some patients remained self-ambulatory in their third decade, whereas others were confined to a wheelchair by their teenage years. The proximal muscles were more severely involved than the distal muscles in all patients, as typical of LGMD, and the lower limbs were considerably more affected than the upper limbs. Mild pseudohypertrophy of the calves was apparent in certain patients. The creatine kinase (CK) levels were elevated in relatively early stages of the disease, with values reaching 3000-40 000 IU/1 (normal value: <190 IU/l). No other biochemical abnormality was found. Muscle biopsies were not available for immunostaining with $\alpha$-dystroglycan antibodies.

All patients complained of back pain or stiffness as one of the prominent symptoms: hyperlordosis, kyphoscoliosis, or spinal fusion was found in all affected individuals. The facial muscles and autonomic functions were spared, with the exception of at least one severely affected patient, who had involvement of facial muscles and difficulties in mastication. Neither primary contractures nor consistent facial dysmorphism were noted in the patients.

Progressive restrictive lung disease, evidence for pulmonary hypertension, and respiratory failure by the early third decade was seen in the more severe cases. No primary cardiac involvement was reported in any of the patients. However, one patient had right heart failure, apparently secondary to pulmonary involvement, and another patient 
died in his third decade of cardiac arrest. Patients were not systematically studied with echocardiography.

The pathological involvement of other organs or functional systems was not reported, and no problems of fertility were recounted in patients. Patients displayed normal cognitive development and intelligence. Brain CT scans of two affected individuals showed no abnormalities; MRI studies were not carried out.

\section{Linkage and haplotype analysis}

Six affected individuals, studied by genome-wide linkage analysis, were all found to be homozygous for the same allele at marker D19S902 (Figure 2). Fine mapping in the region of D19S902, performed on 11 affected individuals and eight healthy individuals, confirmed the association between the disease and the region including markers D19S902 and D19S908. All the affected individuals were homozygous at these two loci, while none of the unaffected individuals were homozygous at both loci. The maximal LOD score obtained was $Z=4.8$ at a recombination fraction $(\theta)$ of 0 , for D19S902. This marker is located approximately 1.07 megabases from FKRP, the gene underlying LGMD2I.

\section{Mutation analysis}

The mutation analysis of a $1.7-\mathrm{kb}$ fragment containing the FKRP coding sequence ${ }^{21}$ revealed a novel missense mutation C160T in exon 4 (the nucleotide number is given with reference to Brockington et al, ${ }^{19}$ and is equivalent to nucleotide 445 in NCBI's 'Homo sapiens FKRP mRNA', gi: 13236527). This mutation causes an alteration in amino acid 54 of the protein from arginine to tryptophan (R54W).

The mutation C160T was found in a homozygous state in eight affected individuals sequenced, by direct sequencing of the fragment of the gene amplified by primers FKRP $1 \mathrm{~F}$ and FKRP 2R (see Figure 3). Six parents (obligate carriers) and one unaffected sibling (a carrier of the affected

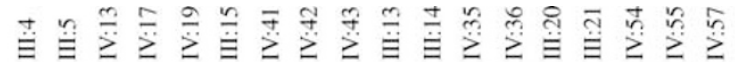

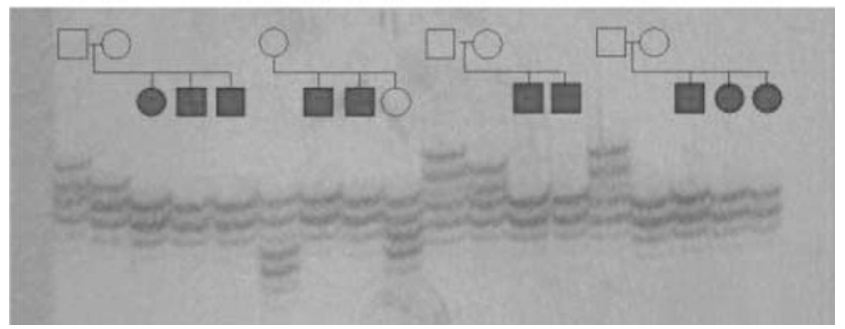

Figure 2 Linkage analysis of marker D19S902. All the affected individuals are homozygous for an identical allele. PCR amplification products were separated on a $6 \%$ polyacrylamide gel and visualized by silver staining. Note: Individual III:21 is uninformative at this marker.

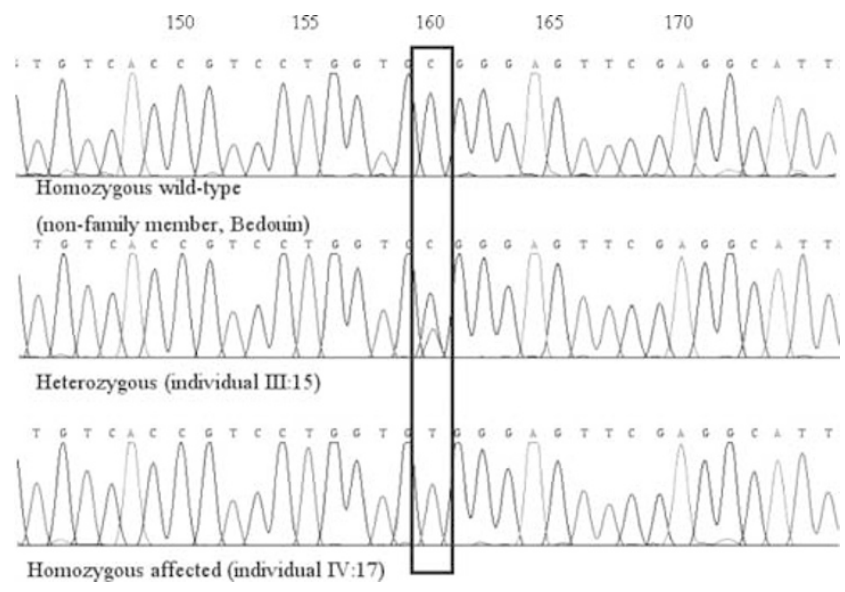

Figure 3 Mutation C160T, analyzed by Chromas software. The upper sequence shows homozygosity for the wild-type allele; the middle sequence shows heterozygosity for the wild-type allele and the point mutation; and the lower sequence shows homozygosity for the mutated allele.

haplotype) were found to be heterozygous for the mutation. The C160T mutation abolishes an AciI restriction site. Based on either differential cleavage by AciI or direct sequencing, 200 alleles of unaffected Bedouin individuals were shown not to harbor the C160T mutation.

A table summarizing the mutations and polymorphisms known to date in the FKRP gene can be found at the website http://www.dmd.nl/fkrp_seqvar.html.

\section{Discussion}

We have identified a novel mutation in FKRP in individuals affected by LGMD2I, in a large consanguineous Bedouin family in Israel. FKRP has recently been implicated in causing this form of LGMD, as well as a more severe allelic variant, congenital muscular dystrophy MDC1C. ${ }^{19}$ MDC1C is characterized by onset in the first weeks of life; severe weakness and wasting of shoulder-girdle muscles; hypertrophy and weakness of the leg, calf, and thigh muscles with an inability to walk; severe restrictive respiratory involvement; variable cardiac involvement; and macroglossia in severe cases. ${ }^{21}$ In distinction, the onset of symptoms in LGMD2I ranges from early childhood to the third decade; patients generally reach ambulation, and loss thereof is widely variable. ${ }^{19}$

The FKRP gene encodes a protein of 495 amino acids that is predicted to be a putative glycosyltransferase. A hydrophobic N-terminal of 33 amino acids, which contains the transmembrane domain, targets and anchors the FKRP protein to the Golgi apparatus. A conserved $\mathrm{D} \times \mathrm{D}$ motif, typical of the active site of glycosyltransferases, is found at amino-acid residues 362-364. FKRP is thought to be required for the post-translational modification of dystroglycan. Consequently, mutations in the putative active site 
of the protein or in the Golgi-targeting sequence are likely to cause mislocalization of the mutant protein in the cell and aberrant processing of dystroglycan, leading to MDC1C or LGMD2I. ${ }^{22}$ In fact, the expression pattern of the $\alpha$-dystroglycan protein was abnormal in all muscle biopsies of LGMD2I patients recently studied. ${ }^{19}$

Recently, abnormal glycosylation of $\alpha$-dystroglycan has been proposed to underlie several inherited muscular dystrophies. $\alpha$-Dystroglycan is a highly glycosylated protein whose sugar components vary in different tissues, thereby controlling its interactions with extracellular matrix components. Fukutin, POMGnT1, and POMT1, the genes defective in Fukuyama congenital muscular dystrophy (OMIM 253800), muscle-eye-brain disease (OMIM 253280), and Walker-Warburg syndrome (OMIM 236670), respectively, have all been hypothesized to encode novel glycosyltransferases involved in the posttranslational processing of $\alpha$-dystroglycan. ${ }^{24-26}$ The product of the FKRP gene, also proposed to participate in the modification of $\alpha$-dystroglycan, lends further support to the notion that abnormal post-translational modification of proteins in the muscle fiber, and not only structural defects in these proteins, is involved in the pathogenesis of certain muscular dystrophies.

A vast majority of the affected individuals studied by Brockington et $a l^{19}$ had an identical C826A (Leu276Ileu) mutation. Those individuals homozygous for this mutation showed a relatively mild phenotype, whereas patients who were compound heterozygotes (with the C826A alteration in one allele) had a more severe phenotype. The mutation did not appear to be associated with a single conserved haplotype, leading to the assumption that it may have arisen independently on multiple events. ${ }^{19}$ The patients in our study had no alteration at nucleotide position 826 or at amino acid 276.

In our current study, all the affected individuals were homozygous for a novel missense mutation C160T. No unaffected individuals in the family were found to be homozygous for the mutation; furthermore, the mutation was not found in a vast number of control alleles. This finding, in conjunction with the significant LOD score of 4.8 at marker D19S902, located approximately 1.07 megabases from FKRP, allows us to deduce a clear association between the novel C160T mutation and the phenotype observed in the affected individuals studied.

The missense mutation causes an alteration in amino acid 54 of the protein from arginine to tryptophan (R54W). This mutation, similar to those mutations previously implicated as causative of LGMD2I, ${ }^{19}$ lies neither in the transmembrane domain nor in the proposed active site of the protein. A search in the databases did not generate a similarity between the mutation site and any conserved motif. Nonetheless, the substitution of an aromatic, hydrophobic amino acid (tryptophan, W) for a positively charged, hydrophilic amino acid (arginine, R) is likely to alter the conformation of the protein.

A challenge that remains in the understanding of LGMD is the variability of the presentation or severity associated with disease caused by a single gene or even a single mutation. ${ }^{27}$ Intrafamilial variability has been reported in autosomal recessive LGMD, particularly in the sarcoglycanopathies, LGMD2C-2F. ${ }^{28}$ Intrafamilial variability in LGMD2I has been mentioned in a consanguineous Tunisian family mapped to $19 \mathrm{q} 13.3,{ }^{18}$ prior to the identification of FKRP as the defective gene. ${ }^{29}$ The availability of small, independent, and mostly nonconsanguineous families in the study that identified FKRP as causative of LGMD2I ${ }^{19}$ did not allow for discussion of a variable phenotype among multiple family members.

Our accessibility to a large consanguineous family with LGMD2I, in the present study, allowed us to appreciate the intrafamilial variability associated with a single mutation in FKRP. Although all the affected individuals were homozygous for the C160T mutation, notable phenotypic variability existed among them, in terms of age of onset (ranging between birth and teenage years), pace of progression, extent of limb weakness and disability, respiratory involvement, and life expectancy. The most consistent findings were severe involvement of the proximal muscles as compared to the distal muscles, and severe involvement of the lower limbs relative to the upper limbs. No association was noted between the phenotype and the gender of the patient. Seemingly, the age of onset in the patients studied was independent of the pace of progression. However, a correlation was noted between the severity of limb weakness and respiratory involvement: patients confined to wheelchairs were those affected by restrictive lung disease.

The novel FKRP mutation identified will enable prenatal diagnosis of embryos at risk for LGMD2I in the consanguineous Bedouin family studied. The intrafamilial phenotypic variability demonstrated by our study, even among siblings, suggests that the clinical heterogeneity of LGMD2I results not only from genetic heterogeneity but also from modifier genes or environmental factors yet to be identified.

Previous studies have associated the congenital onset of muscular dystrophy with MDC1C but not with LGMD2I. We now demonstrate that an identical, novel mutation in the FKRP gene can cause a muscular disease of either a congenital onset or of a later onset within the same family, providing clinical support for the molecular evidence, suggesting that MDC1C and LGMD2I are overlapping ends of one and the same entity.

\section{Acknowledgements}

We thank the Kahn Family Foundation for Humanitarian Support for the generous funding of this project. The research for this paper was carried out, in part, to fulfill the requirements of MD studies of TH. 


\section{References}

1 Moreira ES, Vainzof M, Marie SK, Sertie AL, Zatz M, Passos-Bueno MR: The seventh form of autosomal recessive limb-girdle muscular dystrophy is mapped to 17q11-12. Am J Hum Genet 1997; 61: 151-159.

2 Beckmann JS, Richard I, Hillaire D et al: A gene for limb-girdle muscular dystrophy maps to chromosome 15 by linkage. $C R$ Acad Sci 1991; 312: 141-148.

3 Richard I, Broux O, Allamand V et al: Mutations in the proteolytic enzyme calpain 3 cause limb-girdle muscular dystrophy type $2 \mathrm{~A}$. Cell 1995; 81: 27-40.

4 Bashir R, Strachan T, Keers S et al: A gene for autosomal recessive limb-girdle muscular dystrophy maps to chromosome 2p. Hum Mol Genet 1994; 3: 455-457.

5 McNally EM, Ly CT, Rosenmann $\mathrm{H}$ et al: Splicing mutation in dysferlin produces limb-girdle muscular dystrophy with inflammation. Am J Med Genet 2000; 91: 305-312.

6 Ben Othmane K, Ben Hamida M, Pericak-Vance M et al: Linkage of Tunisian autosomal recessive Duchenne-like muscular dystrophy to the pericentromeric region of chromosome 13q. Nat Genet 1992; 2 : 315 - 317

7 Noguchi S, McNally EM, Ben Othmane K et al: Mutations in the dystrophin-associated protein gamma-sarcoglycan in chromosome 13 muscular dystrophy. Science 1995; 270: 819-821.

8 Roberds SL, Leturcq F, Allamand V et al: Missense mutations in the adhalin gene linked to autosomal recessive muscular dystophy. Cell 1994; 78: 625-633.

9 McNally EM, Yoshida M, Mizuno Y, Ozawa E, Kunkel LM: Human adhalin is alternatively spliced and the gene is located on chromosome 17q21. Proc Natl Acad Sci 1994; 91: 9690-9694.

10 Passos-Bueno MR, Moreira ES, Vainzof M et al: A common missense mutation in the adhalin gene in three unrelated Brazilian families with a relatively mild form of autosomal recessive limbgirdle muscular dystrophy. Hum Mol Genet 1995; 4: 1163-1167.

11 Lim LE, Duclos F, Broux O et al: Beta-sarcoglycan: characterization and role in limb-girdle muscular dystrophy linked to 4q12. Nat Genet 1995; 11: 257-265.

12 Bonnemann CG, Modi R, Noguchi S et al: Beta-sarcoglycan (A3b) mutations cause autosomal recessive muscular dystrophy with loss of the sarcoglycan complex. Nat Genet 1995; 11: 266-273.

13 Passos-Bueno MR, Moreira ES, Vainzof M, Marie SK, Zatz M: Linkage analysis in autosomal recessive limb-girdle muscular dystrophy (AR LGMD) maps a sixth form to 5q33-34 (LGMD2F) and indicates that there is at least one more subtype of AR LGMD. Hum Mol Genet 1996; 5: 815-820.

14 Nigro V, Moreira ES, Piluso G et al: Autosomal recessive limbgirdle muscular dystrophy, LGMD2F, is caused by a mutation in the delta-sarcoglycan gene. Nat Genet 1996; 14: 195-198.

15 Moreira ES, Wiltshire TJ, Faulkner G et al: Limb-girdle muscular dystrophy type $2 \mathrm{G}$ is caused by mutations in the gene encoding the sarcomeric protein telethonin. Nat Genet 2000; 24: 163-166.
16 Weiler T, Greenberg CR, Zelinski T et al: A gene for autosomal recessive limb-girdle muscular dystrophy in Manitoba Hutterites maps to chromosome region 9q31-q33: evidence for another limb-girdle muscular dystrophy locus. Am J Hum Genet 1998; 63: $140-147$.

17 Frosk P, Weiler T, Nylen E et al: Limb-girdle muscular dystrophy type $2 \mathrm{H}$ associated with mutation in TRIM32, a putative E3-ubiquitin-ligase gene. Am J Hum Genet 2002; 70: 663-672.

18 Driss A, Amouri R, Ben Hamida C et al: A new locus for autosomal recessive limb-girdle muscular dystrophy in a large consanguineous Tunisian family maps to chromosome $19 \mathrm{q} 13.3$. Neuromuscul Disord 2000; 10: 240-246.

19 Brockington M, Yuva Y, Prandini $\mathrm{P}$ et al: Mutations in the fukutin-related protein gene (FKRP) identify limb girdle muscular dystrophy 2I as a milder allelic variant of congenital muscular dystrophy MDC1C. Hum Mol Genet 2001; 10: $2851-2859$.

20 Haravuori H, Vihola A, Straub V et al: Secondary calpain3 deficiency in 2q-linked muscular dystrophy: titin is the candidate gene. Neurology 2001; 56: 869-877.

21 Brockington M, Blake DJ, Prandini P et al: Mutations in the fukutin-related protein gene (FKRP) cause a form of congenital muscular dystrophy with secondary laminin alpha2 deficiency and abnormal glycosylation of alpha-dystroglycan. Am J Hum Genet 2001; 69: 1198-1209.

22 Esapa CT, Benson MA, Schroder JE et al: Functional requirements for fukutin-related protein in the Golgi apparatus. Hum Mol Genet 2002; 11: 3319-3331.

23 Mykytyn K, Braun T, Carmi R et al: Identification of the gene that, when mutated, causes the human obesity syndrome BBS4. Nat Genet 2001; 28: 188-191.

24 Muntoni F, Brockington M, Blake DJ, Torelli S, Brown SC: Defective glycosylation in muscular dystrophies. Lancet 2002; 360: 1419-1421.

25 Michele DE, Barresi R, Kanagawa $\mathrm{M}$ et al: Post-translational disruption of dystroglycan-ligand interactions in congenital muscular dystrophies. Nature 2002; 418: 417-422.

26 Beltran-Valero de Bernabe D, Currier S, Steinbrecher A et al: Mutations in the $O$-mannosyltransferase gene POMT1 give rise to the severe neuronal migration disorder Walker-Warburg syndrome. Am J Hum Genet 2002; 71: 1033-1043.

27 Bushby KMD, Beckmann JS: The 105th ENMC sponsored workshop: pathogenesis in the non-sarcoglycan limb-girdle muscular dystrophies, Naarden, April 12-14, 2002. Neuromuscul Disord 2003; 13: 80-90.

28 Bushby KMD: Making sense of the limb-girdle muscular dystrophies. Brain 1999; 122: 1403-1420.

29 Brockington M, Blake DJ, Brown SC, Muntoni F: The gene for a novel glycosyltransferase is mutated in congenital muscular dystrophy MDC1C and limb girdle muscular dystrophy 2I. Neuromusc Disord 2002; 12: 233-234. 